\title{
Inhibition of the acrosome reaction by trypsin inhibitors and prevention of penetration of spermatozoa through the human zona pellucida
}

\author{
M. Llanos ${ }^{1}$, P. Vigil ${ }^{2}$, A. M. Salgado ${ }^{2}$ and P. Morales ${ }^{2 *}$ \\ ${ }^{1}$ Unit of Reproductive Biology, INTA, University of Chile; and ${ }^{2}$ Unit of Reproduction and \\ Development, Faculty of Biological Sciences, P. Catholic University of Chile, PO Box 114-D, \\ Santiago, Chile
}

\begin{abstract}
In this study we evaluated the effect of several trypsin inhibitors ( $p$-aminobenzamidine: $\mathrm{pAB}$; $N$ - $\alpha$ - $p$-tosyl-L-lysine-chloromethyl-ketone: TLCK and $p$-nitrophenyl- $p^{\prime}$-guanidino-benzoate: NPGB) on sperm binding and penetration of the human zona pellucida. Motile spermatozoa, selected by a two-step Percoll gradient, were incubated at $1 \times 10^{7}$ cells ml ${ }^{-1}$ at $37^{\circ} \mathrm{C}$ and in $5 \% \mathrm{CO}_{2}$ for $4.5 \mathrm{~h}$. This was followed by the addition of $1 \mathrm{mmol} \mathrm{pAB} 1^{-1}$ or phosphatebuffered saline (control) for $30 \mathrm{~min}$. Three to four non-viable human oocytes were then added to each sperm suspension and incubated for $3 \mathrm{~h}$. The numbers of spermatozoa bound to the human zona pellucida and in the perivitelline space were determined by phase contrast microscopy. The results showed that $\mathrm{PAB}$ significantly inhibited zona penetration by spermatozoa $(56 \pm 8 \%$ oocytes penetrated, control versus $0 \pm 0 \%$ oocytes penetrated, pAB, mean \pm SEM), without modifying spermatozoa-zona pellucida binding. The inhibition of zona penetration was due to a block of the acrosome reaction normally induced by the human zona pellucida. In separate experiments, sperm suspensions pretreated with $1 \mathrm{mmol}$

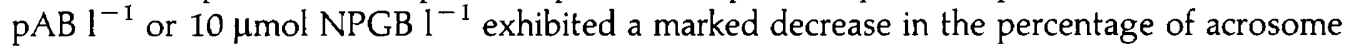
reactions on the zona surface ( $85 \pm 4 \%$ and $76 \pm 3 \%$ inhibition, respectively). In addition, the inhibitors prevented the acrosome reaction induced by human follicular fluid (percentage of acrosome-reacted spermatozoa: control $8 \pm 2$; follicular fluid $25 \pm 3$; pAB $6 \pm 2$; NPGB $8 \pm 1$; TLCK $12 \pm 2$ ). Electron microscope studies suggested a significant inhibition of the membrane fusion events of the acrosome reaction in the inhibitor-treated spermatozoa. These results are the first to show that trypsin inhibitors block sperm penetration of the human zona pellucida owing to an inhibition of the acrosome reaction. In addition, they suggest a role for a trypsin-like enzyme during the acrosome reaction of human spermatozoa.
\end{abstract}

\section{Introduction}

The interaction between spermatozoa and the zona pellucida is a crucial step during mammalian fertilization (Yanagimachi, 1988). This interaction involves several levels. First, the spermatozoa attach to the zona pellucida. This primary attachment is performed by either acrosome-intact or acrosomereacted spermatozoa. In the mouse, however, only acrosomeintact spermatozoa can initiate binding to the zona pellucida (Florman and Storey, 1982; Saling and Storey, 1979). The initial binding is followed by either the zona-induced acrosome reaction or by secondary sperm binding and penetration of the zona pellucida (Yanagimachi, 1988). It is generally accepted that spermatozoa must be acrosome reacted to complete penetration of the zona. However, the exact site where the acrosome reaction occurs in vivo has not been determined. Various components of the zona pellucida involved in gamete

*Reprint requests.

Received 3 February 1992 interaction have been identified and characterized. In the mouse, the zona pellucida is composed of three glycoproteins termed $\mathrm{ZP1}, \mathrm{ZP2}$ and ZP3. ZP3 is responsible for binding acrosomeintact spermatozoa to the zona pellucida and for stimulating the acrosome reaction (Wassarman et al., 1989). ZP2 has been implicated in the secondary binding of acrosome-reacted spermatozoa to the zona (Bleil et al., 1988). It has been suggested that a trypsin-like enzyme of spermatozoa could participate in several of these processes (Saling, 1981; Dravland et al., 1984; Bleil et al., 1988). For instance, there is evidence that sperm penetration through the zona pellucida involves localized proteolysis (McRorie and Williams, 1974). Before the morphologically visible human sperm acrosome reaction begins, limited amounts of activated, immunoreactive acrosin are mobilized to the sperm surface. The enzyme was detected on the plasma membrane covering the acrosomal area (Tesarik et al., 1988, 1990). This finding supports a role for acrosin in spermatozoazona pellucida initial binding and in the early biochemical events of the human sperm acrocome reaction (De Jonge et al., 1989). In non-human species, acrosin has been associated with 
the acrosome reaction, participating either in the dispersal of the acrosomal matrix or in the membrane events of the acrosome reaction, or in both (Shams-Borhan and Harrison, 1981; Dravland et al., 1984). It has also been suggested that a trypsin-like enzyme may be involved in the binding of mouse spermatozoa to the zona pellucida (Saling, 1981; Benau and Storey, 1987).

In the present study, we investigated the influence of several low molecular weight trypsin inhibitors on the interaction of human spermatozoa with zona pellucida. We performed experiments to test the effect of these inhibitors on the ability of spermatozoa to bind to the human zona pellucida, to undergo the acrosome reaction on the zona surface, to penetrate the human zona pellucida, and to undergo the acrosome reaction upon stimulation with human follicular fluid. Our results suggest a role for a trypsin-like enzyme in the mechanism by which human spermatozoa undergo the acrosome reaction, either induced by human zona pellucida or human follicular fluid. Part of these results has been presented elsewhere (Morales et al., 1990).

\section{Materials and Methods}

\section{Sperm preparation}

Normal semen samples, obtained for diagnostic purposes at the Center for the Study of Reproductive Biology (CEBRE), of the P. Catholic University of Chile, were used. The specimens were obtained after two to four days of sexual abstinence and were allowed to liquefy for $30-60 \mathrm{~min}$ at room temperature. The samples were used within $1 \mathrm{~h}$ of collection. Motile spermatozoa were selected by centrifugation through a twostep Percoll gradient (Yudin et al., 1988). The two steps of the Percoll gradient were $80 \%$ in the lower layer and $40 \%$ in the upper layer. Briefly, 1-2 ml aliquots of semen were layered over the upper layer of the Percoll gradient and then centrifuged for $20 \mathrm{~min}$ at $300 \mathrm{~g}$. The pellet was washed twice by dilution with $10 \mathrm{ml}$ of modified Tyrode's medium (Yudin et al., 1988) supplemented with $0.3 \%$ bovine serum albumin (BSA, Fraction V, Sigma Chemical Co., St Louis, MO). The sperm suspension was then centrifuged for $10 \mathrm{~min}$ at $300 \mathrm{~g}$. The final pellet was diluted in modified Tyrode's medium supplemented with $2.6 \%$ BSA. Sperm suspensions were adjusted to $1 \times 10^{7}$ cells $\mathrm{ml}^{-1}$ and incubated in $1 \mathrm{ml}$ aliquots for various periods at $37^{\circ} \mathrm{C}$ in $5 \% \mathrm{CO}_{2}, 95 \%$ air.

\section{Collection of human oocytes}

Human oocytes were dissected from ovarian tissue obtained from cadavers, and stored at $-80^{\circ} \mathrm{C}$ as previously described (Cross et al., 1988). After thawing, the oocytes were freed of remaining cumulus cells by passing them through a narrow bore pipette. As a result of freezing and thawing, these oocytes were not viable.

\section{Collection of follicular fluid}

The fluids used in this study were collected from preovulatory follicles after gonadotrophin stimulation and were kept frozen in aliquots at $-20^{\circ} \mathrm{C}$ until use. The human follicular fluid used here was selected for its effectiveness in inducing acrosome reactions in previous experiments (Morales et al., 1992).

\section{Effect of inhibitors on the penetration of zona pellucida by spermatozoa}

The effect of protease inhibitors on penetration of zona pellucida by spermatozoa was determined by incubating sperm suspensions for 5 or $20 \mathrm{~h}$ and then treating with $1 \mathrm{mmol} p$ aminobenzamidine $\mathrm{l}^{-1}(\mathrm{pAB})$ or with phosphate-buffered saline for $30 \mathrm{~min}$. Subsequently, four human oocytes were added to each sperm suspension and the gametes were coincubated for an additional $3 \mathrm{~h}$. The number of spermatozoa attached to the human zona pellucida was determined using phase contrast microscopy (the terms 'attached' and 'bound' will be used interchangeably to refer to any adherent spermatozoa, Cross et al., 1988). Zona penetration was evaluated by aspirating oocytes in and out of a small-bore pipette several times to remove excess spermatozoa and they were then mounted between a glass slide and cover slip supported by four pillars of Vaseline-paraffin. Each human oocyte was repeatedly rolled between the slide and cover glass and the number of spermatozoa in the perivitelline space was determined (Lambert et al., 1985).

\section{Effect of inhibitors on the sperm acrosome reaction}

The effect of protease inhibitors on the human sperm acrosome reaction was evaluated using intact zonae pellucidae or preovulatory human follicular fluid to induce the acrosome reaction, as described below. $\mathrm{pAB}$ and $\mathrm{N \alpha}$-p-tosyl-L-lysinechloromethyl-ketone (TLCK) were dissolved in phosphatebuffered saline, $\mathrm{pH}$ 7.3. $p$-Nitrophenyl- $p^{\prime}$-guanidino-benzoate (NPGB) was dissolved in dimethylsulfoxide. Inhibitor additions were made by adding $2 \mu \mathrm{l}$ of the particular compound to $198 \mu \mathrm{l}$ of capacitated spermatozoa; final inhibitor concentrations were

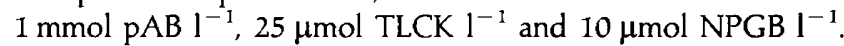
The final concentration of dimethylsulfoxide in sperm suspensions was $0.1 \%(\mathrm{v} / \mathrm{v})$. The concentrations of inhibitors used in the present study were the highest possible that did not inhibit sperm motility. The inhibitors were purchased from Sigma Chemical Co.

\section{Acrosome reactions induced by human zona pellucida}

Spermatozoa were incubated for $4.5 \mathrm{~h}$ and, separate aliquots were then incubated with $1 \mathrm{mmol} \mathrm{pAB} \mathrm{l}^{-1}$ or $10 \mu \mathrm{mol} \mathrm{NPGB}$ $1^{-1}$ for $30 \mathrm{~min}$. Sperm suspensions pretreated with NPGB were washed and resuspended in fresh, inhibitor-free media before adding the zonae pellucidae. Control sperm suspensions were incubated with the appropriate solvents. Four human oocytes were then added to each aliquot and incubated for a further $30 \mathrm{~min}$. The oocytes with adhered spermatozoa were then fixed in ethanol and the acrosomal status of the bound spermatozoa was determined by indirect immunofluorescence using a polyclonal antisperm antiserum (Cross et al., 1986; Morales et al., 1989).

\section{Acrosome reactions induced by human follicular fluid}

Spermatozoa were incubated for $20 \mathrm{~h}$ and separate aliquots

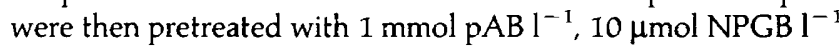


or $25 \mu \mathrm{mol} \mathrm{TLCK} \mathrm{l}^{-1}$ for $30 \mathrm{~min}$. Sperm suspensions pretreated with NPGB or TLCK were always washed and resuspended in fresh, inhibitor-free media before adding the human follicular fluid. Control sperm suspensions were incubated with the appropriate solvents. The spermatozoa were then treated with $20 \%$ human follicular fluid $(\mathrm{v} / \mathrm{v})$ for a further $15 \mathrm{~min}$. The acrosome reaction was detected using fluoresceinated Pisum sativum agglutinin (Vector Laboratories, Inc., Burlingame, CA) as described previously (Cross et al., 1986). Pisum sativum agglutinin and antisperm antiserum provide equivalent assessments of acrosomal status (Cross et al., 1986, 1988), but Pisum satioum agglutinin was used for sperm suspensions because the method is simpler and more rapid. The antiserum was used for spermatozoa on the zona pellucida because Pisum sativum agglutinin binds to the zona pellucida and obscures the pattern of sperm labelling.

\section{Electron microscopy}

In other experiments, spermatozoa pretreated with inhibitors and then with human follicular fluid were processed for transmission electron microscopy. The spermatozoa were fixed in a solution of $3 \%$ glutaraldehyde prepared in albumin-free cacodylate buffer $\left(0.25 \mathrm{~mol} \mathrm{l}^{-1}\right) \mathrm{pH} 7.4$. The samples were then dehydrated in a series of increasing concentrations of acetone and embedded in Spurr's embedding medium Ted Pella, Inc., Redding, CA. Ultrathin sections were obtained in the MT 2B Porter Blum ultramicrotome. The grids with sections were stained with uranyl acetate and lead citrate. The numbers of acrosome-intact and acrosome-reacted spermatozoa were evaluated using a Siemens I-AKV transmission electron microscope. One hundred spermatozoa were scored in each group.

\section{Results}

In the control group, about $60 \%$ of the oocytes were penetrated by one or more spermatozoa in the perivitelline space $(2.1 \pm 0.3$ spermatozoa in the perivitelline space, mean $\pm \mathrm{SEM}$, $n=26$ penetrated oocytes). The ability of the spermatozoa to penetrate the human zona pellucida was totally inhibited by $1 \mathrm{mmol} \mathrm{pAB}^{-1}$ (Fig. 1). There were no oocytes with spermatozoa in the perivitelline space in the $\mathrm{pAB}$-treated group. This was the case whether the spermatozoa were incubated for $5 \mathrm{~h}$ or for $20 \mathrm{~h}$. The number of spermatozoa bound to the human zona pellucida was unaffected by the presence of the inhibitor (120 \pm 25 and $100 \pm 30$ sperm bound per zona, control and pAB-treated cells, respectively, $n=11$ ). The inhibition of sperm passage through the human zona pellucida could be due to either an inhibition of the enzyme responsible for local digestion of the zona or an inhibition of the acrosome reaction itself. The latter was tested by determining the percentage of spermatozoa that could undergo the acrosome reaction on the surface of the human zona pellucida, in the presence or absence of trypsin inhibitors. After $30 \mathrm{~min}$ of coincubation of the gametes, the percentage of acrosome reaction among the zonabound spermatozoa was between 40 and $50 \%$ in the control groups (Fig. 2). However, when the cells were pretreated with the inhibitors, the percentage of acrosome-reacted spermatozoa

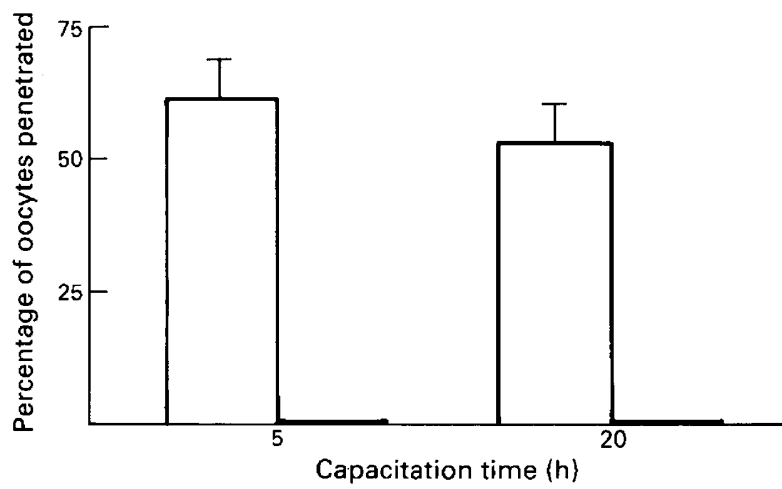

Fig. 1. Effect of $1 \mathrm{mmol} p$-aminobenzamidine $\mathrm{l}^{-1}(\mathrm{pAB})$ on the ability of human spermatozoa to penetrate the human zona pellucida. Results are means \pm SEM of seven experiments carried out after $5 \mathrm{~h}$ of sperm capacitation and four experiments carried out after $20 \mathrm{~h}$ of sperm capacitation. Four oocytes were used in each experiment. ( $\square$ ) Control and $(\boldsymbol{\square})$ pAB-treated groups.

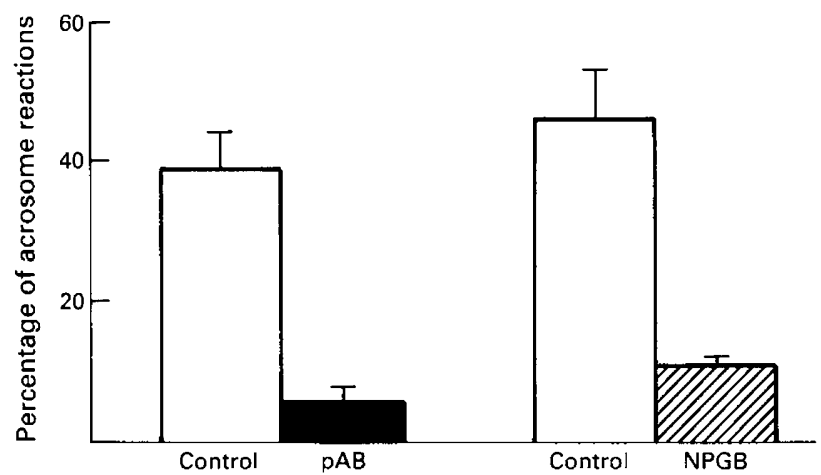

Fig. 2. Effect of 1 mmol $p$-aminobenzamidine $\mathrm{l}^{-1}(\mathrm{pAB})$ and $10 \mu \mathrm{mol}$ $p$-nitrophenyl- $p^{\prime}$-guanidino-benzoate (NPGB) on the ability of human spermatozoa to undergo the acrosome reaction on the zona surface. Spermatozoa pretreated with NPGB were washed and resuspended in inhibitor-free media before adding the oocytes. Results are means \pm SEM of three experiments with each inhibitor. Four oocytes were used in each experiment.

on the zona surface was dramatically decreased $(85 \pm 4 \%$ and $76 \pm 3 \%$ inhibition for $\mathrm{pAB}$ - and NPGB-treated cells, respectively, $n=3$ ). Again, the number of spermatozoa bound to the human zona pellucida was not different between the control and the treated groups ( $\mathrm{pAB}$-treated: $106 \pm 18$ spermatozoa bound per zona; NPGB-treated: $82 \pm 18$ spermatozoa bound per zona; control: $91 \pm 20$ spermatozoa bound per zona).

In additional experiments, we tested the ability of the various inhibitors to prevent the acrosome reaction induced by treatment with human follicular fluid (Fig. 3). Treatment with human follicular fluid induced a 3.5 -fold increase in the percentage of acrosome-reacted spermatozoa, in comparison to the control group. Spermatozoa pretreated with $\mathrm{pAB}, \mathrm{NPGB}$ or TLCK before the addition of human follicular fluid showed a $93 \pm 7$, $90 \pm 6$ and $76 \pm 11 \%$ inhibition of the acrosome reaction, respectively, in comparison with the human follicular fluid group 


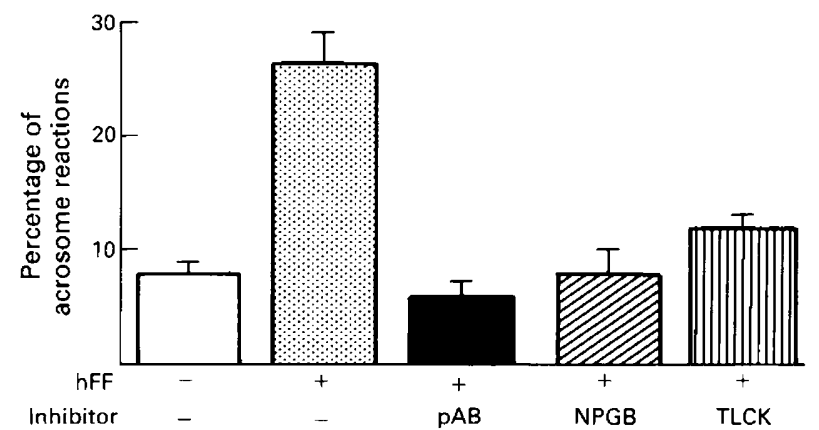

Fig. 3. Effect of $1 \mathrm{mmol} p$-aminobenzamidine $\mathrm{L}^{-1}$ (pAB), $10 \mu \mathrm{mol}$ $p$-nitrophenyl- $p^{\prime}$-guanidino-benzoate $\mathrm{I}^{-1}$ (NPGB), and $25 \mu \mathrm{mol} N \alpha-p$ tosyl-L-lysine-chloromethyl-ketone $\mathrm{I}^{-1}$ (TLCK) on the ability of human spermatozoa to undergo the acrosome reaction upon stimulus with human follicular fluid (hFF). All the spermatozoa were treated with $20 \%$ human follicular fluid for $15 \mathrm{~min}$, except the control group which received media alone. The various inhibitors were then added to the sperm suspensions. Spermatozoa pretreated with NPGB or TLCK were washed and resuspended in inhibitor-free media before adding the human follicular fluid. Results are means \pm SEM of eight experiments.

(Fig. 3). We did not detect intermediate stages of the acrosome reaction in the inhibitor-treated groups (Fig. 4). The percentage of acrosome-reacted spermatozoa in the group treated with human follicular fluid was $48 \%$, in the pAB-treated groups was $16 \%$, and in the NPGB-treated group was $18 \%$. The basal percentage of acrosome reacted spermatozoa was between 8 and $12 \%$.

\section{Discussion}

This study is the first to show that trypsin inhibitors prevent human sperm penetration through the zona pellucida of human oocytes. We suggest that the inhibition of zona penetration was caused by a block of the acrosome reaction induced by human zona pellucida. The ability of the sperm cells to bind to the human zona pellucida was not affected by the inhibitors. In addition, the inhibitors used in this study were able to block the human follicular fluid-induced acrosome reaction. These observations support a role for an endogenous trypsin-like enzyme from spermatozoa in the human sperm acrosome reaction, induced by either human follicular fluid or human zona pellucida. Our observations, however, do not support a role for a trypsin-like enzyme from spermatozoa in the primary spermatozoa-zona binding, as reported for mouse gametes (Saling, 1981; Benau and Storey, 1987).

It is not known at what level this trypsin-like enzyme acts during the sperm acrosome reaction. It has been suggested, for non-human species, that a trypsin-like activity may be involved in the membrane events of the acrosome reaction (Dravland et al., 1984), or in the dispersal of the acrosomal matrix (ShamsBorhan and Harrison, 1981; Perreault et al, 1982). Pillai and Meizel (1991) showed that trypsin inhibitors block the membrane events of the human sperm acrosome reaction. Our electron microscopy results also suggest that the inhibitors blocked the membrane fusion events of the acrosome reaction. However, the possibility that dispersal of the acrosomal matrix was also prevented by the inhibitors cannot be ruled out by the present data.

It has long been recognized that a trypsin-like enzyme from spermatozoa has a role in zona pellucida penetration, through a limited proteolysis of the zona pellucida glycoproteins (Fraser, 1982; Dunbar et al., 1985). The sperm trypsin-like enzyme implicated in gamete binding and recognition has been suggested to be acrosin, or its zymogen form proacrosin (Töpfer-Petersen and Henschen, 1987; Jones et al., 1988). However, it is not clear whether the enzyme or zymogen would participate in the primary or in the secondary sperm binding to the zona, or in both. In the mouse, it was suggested that acrosin may be involved in both types of binding (Saling, 1981; Benau and Storey, 1987; Bleil et al., 1988). It has been also shown that before the morphological events of the acrosome reaction, immunoreactive acrosin appears on the surface of the plasma membrane overlying the human sperm acrosome (Tesarik et al., $1988,1990)$. Thus, the authors proposed a role for acrosin in the primary binding of human spermatozoa with zona pellucida. Our results suggest that a trypsin-like enzyme may be involved in the human sperm acrosome reaction, but not in the primary binding between spermatozoa and human zona pellucida. The observation that treatment of spermatozoa with the inhibitors pAB or NPGB did not decrease the number of cells bound to the zona supports this assumption. However, we cannot draw any conclusion regarding a role for this trypsin-like enzyme during the secondary binding of spermatozoa to the human zona pellucida.

Although the identity of this human sperm trypsin-like enzyme is not clear, the best known mammalian sperm trypsinlike enzyme is acrosin. In this study, we used various low molecular weight serine protease inhibitors, known to inhibit trypsin-like enzymes through different mechanisms (MaresGuia and Shaw, 1965; Chase and Shaw, 1970). The most probable action of these inhibitors in the sperm cell could therefore be related to proacrosin or acrosin inhibition (De Jonge et al., 1989). Indeed, during extraction of this enzyme from spermatozoa it was shown that these inhibitors prevented the conversion of proacrosin to acrosin (Goodpasture et al., 1981). It has also been shown that inhibitors of this type at concentrations above $10 \mu \mathrm{mol} 1^{-1}$ block acrosin activity in whole human spermatozoa (Kaminski et al., 1987). Nevertheless, the possibility that these inhibitors could be acting on hydrolytic enzymes other than acrosin cannot be excluded. Somatic cell phospholipase $A_{2}$ can be a potential target for serine protease inhibitors (Hesse et al., 1984) and phospholipase $A_{2}$ has also been implicated in the process of acrosome reaction (Llanos et al., 1982). However, a concentration of NPGB as high as $1 \mathrm{mmol} \mathrm{l}^{-1}$ did not inhibit this enzyme (Anderson et al., 1988). In our study the low inhibitor concentrations used should therefore have precluded any significant effect on phospholipase $\mathrm{A}_{2}$.

Several investigators, using species other than humans, have proposed a role for a trypsin-like enzyme from spermatozoa in the acrosome reaction (Meizel and Lui, 1976; Dravland et al., 1984). Our results with human spermatozoa suggest that human zona pellucida and follicular fluid induce the human sperm acrosome reaction through a common mechanism, mediated by a trypsin-like activity. It has been shown that the acrosome reaction induced by human follicular fluid involves a 

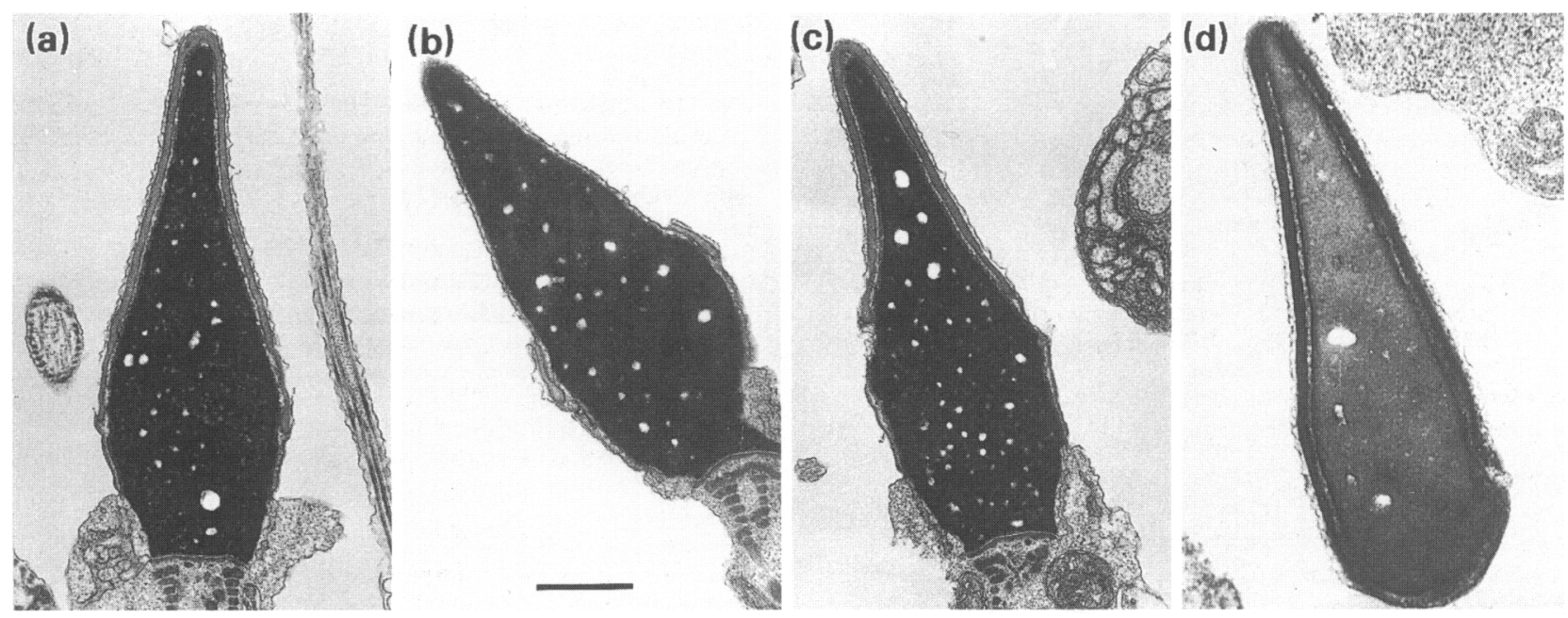

Fig. 4. Electron micrographs of human spermatozoa showing: (a) an acrosome-intact cell in the control group; (b) a typical acrosome-reacted spermatozoa from the human follicular fluid-treated group; (c) a representative, acrosome-intact spermatozoa from the human follicular fluid plus $p$-aminobenzamidine (pAB) group; (d) a representative, acrosome-intact spermatozoa from the human follicular fluid plus $p$-nitrophenyl$p^{\prime}$-guanidino-benzoate (NPGB) group. The percentage of acrosome-reacted spermatozoa was $10 \%$ in the control group, $48 \%$ in the human follicular fluid-treated group, $16 \%$ in the human follicular fluid plus pAB group, and $18 \%$ in the human follicular fluid plus NPGB group. The bar represents $1 \mu \mathrm{m}$.

rapid and transient influx of extracellular calcium into human spermatozoa (Thomas and Meizel, 1988; Blackmore et al., 1990). Similar findings have been reported during the mouse and bovine zona pellucida-induced acrosome reaction (Lee and Storey, 1988; Florman et al., 1989). Pillai and Meizel (1991) reported that trypsin inhibitors block the progesterone-induced acrosome reaction by preventing calcium influx, suggesting a role for a trypsin-like enzyme in increasing intracellular calcium of spermatozoa during the early events of the acrosome reaction. These findings and those of Tesarik et al. (1990), which demonstrate the presence of active acrosin on the surface of the human spermatozoa before the acrosome reaction, support a role for acrosin before stimulation of calcium entry. We propose that a trypsin-like enzyme, presumably acrosin, is playing a key role in the molecular events of the human sperm acrosome reaction. The enzyme could be activating other systems through proteolytic cleavages and thus committing the capacitated spermatozoa to the irreversible morphological events of the acrosome reaction.

We thank H. B. Croxatto for his help and encouragement throughout this research. This study was supported by RF grants GA PS 9110 and FONDECYT $0823 / 90$ to PM.

\section{References}

Anderson RA, Johnson SK and Zaneveld LJD (1988) Isolation and partial characterization of PLA from human spermatozoa Biology of Reproduction 38 (Supplement 1) 173

Benau DA and Storey BT (1987) Characterization of the mouse sperm plasma membrane zona-binding site sensitive to trypsin inhibitors Biology of Reproduction 36 282-292

Blackmore P, Beebe SJ, Danforth DR and Alexander N (1990) Progesterone and $17 \alpha$-hydroxy-progesterone. Novel stimulators of calcium influx in human sperm Journal of Biological Chemistry 265 1376-1380
Bleil JD, Greve JM and Wassarman PM (1988) Identification of a secondary sperm receptor in the mouse egg zona pellucida. Role in maintenance of binding of acrosome-reacted sperm Developmental Biology 128 376-385

Chase T and Shaw E (1970) Titration of trypsin, plasma and thrombin with $p$-nitrophenyl $p^{\prime}$-guanidinobenzoate $\mathrm{HCl}$. In Methods in Enzymology, pp. 20-27 Eds GE Perlman and L Lorand. Vol. 19. Academic Press, New York

Cross NL, Morales P, Overstreet JW and Hanson FW (1986) Two simple methods for detecting acrosome reacted human sperm Gamete Research 15 213-226

Cross NL, Morales P, Overstreet JW and Hanson FW (1988) Induction of the acrosome reaction by human zona pellucida Biology of Reproduction $\mathbf{3 8}$ 235-244

De Jonge CJ, Mack SR and Zaneveld LJD (1989) Inhibition of the human sperm acrosome reaction by proteinase inhibitors Gamete Research 23 387-397

Dravland JE, Llanos MN, Munn RJ and Meizel S (1984) Evidence for the involvement of a sperm trypsin-like enzyme in the membrane events of the hamster sperm acrosome reaction Journal of Experimental Zoology 232 117-128

Dunbar BS, Dudkiewicz JW and Bundman DS (1985) Proteolysis of specific porcine zona pellucida glycoproteins by boar acrosin Biology of Reproduction $32619-630$

Florman HM and Storey BT (1982) Mouse gamete interactions: the zona pellucida is the site of the acrosome reaction leading to fertilization in vitro Developmental Biology 91 121-130

Florman HM, Tombes RM, First NL and Babcock DF (1989) An adhesion associated agonist from the zona pellucida activates $\mathrm{G}$-protein promoted elevation in $\mathrm{Ca}^{2+}$ and $\mathrm{pH}$ that mediate mammalian sperm acrosomal exocytosis Developmental Biology 135 133-146

Fraser L (1982) p-Aminobenzamidine, an acrosin inhibitor, inhibits mouse sperm penetration of the zona pellucida but not the acrosome reaction Journal of Reproduction and Fertility 65 185-194

Goodpasture JC, Polakoski KL and Zaneveld LJD (1981) Acrosin, proacrosin and acrosin inhibitor of human spermatozoa. Extraction, quantitation, and stability Journal of Andrology 1 16-27

Hesse B, Lankisch PG and Kunze H (1984) Effect of the serine protease inhibitor gabexate mesilate on purified pancreatic phospholipase $A_{2}$ Pharmaceutical Research Communications 16 637-645

Jones R, Brown CR and Lancaster RT (1988) Carbohydrate-binding properties of boar sperm proacrosin and assessment of its role in sperm-egg recognition and adhesion during fertilization Development 102 781-792

Kaminski JM, Smith D, Reid DS, Kennedy W, Jeyendran RS and Zaneveld LJD (1987) Effect of aryl 4-guanidinobenzoates on the acrosin activity of human spermatozoa Biology of Reproduction 36 1170-1176 
Lambert H, Overstreet JW, Morales P, Hanson FW and Yanagimachi R (1985) Sperm capacitation in the female reproductive tract Fertility and Sterility $\mathbf{4 3}$ 325-327

Lee MA and Storey BT (1988) Influx of $\mathrm{Ca}^{++}$is the primary reaction mediating the first stage of the zona induced acrosome reaction in mouse spermatozoa Biology of Reproduction 38 (Supplement 1), 93

Llanos MN, Lui CW and Meizel S (1982) Studies of phospholipase $A_{2}$ related to the hamster sperm acrosome reaction Journal of Experimental Zoology 221 117-127

McRorie RA and Williams WL (1974) Biochemistry of mammalian fertilization Annual Review of Biochemistry 43 777-803

Mares-Guia M and Shaw E (1965) Studies on the active center of trypsin Joumal of Biological Chemistry 240 1579-1585

Meizel S and Lui CW (1976) Evidence for the role of a trypsin-like enzyme in the hamster sperm acrosome reaction Joumal of Experimental Zoology 195 137-144

Morales P, Cross NL, Overstreet JW and Hanson FW (1989) Acrosome intact and acrosome reacted human sperm can initiate binding to the zona pellucida Developmental Biology 133 385-392

Morales P, Vigil P and Llanos M (1990) Role of acrosin in the human sperm acrosome reaction Joumal of Andrology $12 \mathrm{P} 41$

Morales P, Llanos M, Gutierrez G, Kohen P, Vigil P and Vantman D (1992) The acrosome reaction inducing activity of individual human follicular fluid samples is highly variable and is related to the steroid content Human Reproduction 7 646-651

Perreault SD, Zirkin BR and Rogers BJ (1982) Effect of trypsin inhibitors on acrosome reaction of guinea pig spermatozoa Biology of Reproduction 26 $343-351$

Pillai MC and Meizel S (1991) Trypsin inhibitors prevent the progesteroneinitiated increase in intracellular calcium required for the human sperm acrosome reaction Joumal of Experimental Zoology 258 384-393
Saling PM (1981) Involvement of trypsin-like activity in binding of mouse spermatozoa to zonae pellucidae Proceeding of the National Academy of Sciences USA 78 6231-6235

Saling PM and Storey BT (1979) Mouse gamete interactions during fertilization in vitro. Chlortetracycline as a fluorescent probe for the mouse sperm acrosome reaction Journal of Cellular Biology 83 544-554.

Shams-Borham G and Harrison RAP (1981) Production, characterization, and use of ionophore-induced, calcium-dependent acrosome reaction in ram spermatozoa Gamete Research 4 407-432

Tesarik J, Drahorad J and Peknicova J (1988) Subcellular immunochemical localization of acrosin in human spermatozoa during the acrosome reaction and zona pellucida penetration Fertility and Sterility 50 133-141

Tesarik J, Drahorad J, Testar J and Mendoza C (1990) Acrosin activation follows its surface exposure and precedes membrane fusion in human sperm acrosome reaction Development 110 39I-400

Thomas $P$ and Meizel S (1988) An influx of extracellular calcium is required for initiation of the human sperm acrosome reaction induced by human follicular fluid Gamete Research 20 397-411

Töpfer-Petersen E and Henschen A (1987) Acrosin shows zona and fucose binding, novel properties for a serine proteinase FEBS Letters $22638-42$

Wassarman PM, Bleil JD, Fimiani C, Florman HM, Greve JM, Kinloch R, Moller C, Mortillo S, Roller RJ, Salzmann GS and Vazquez M (1989) The mouse egg receptor for sperm: a multifunctional zona pellucida glycoprotein. In The Mammalian Eg8 Coat: Structure and Function, pp 18-37 Ed. J Dietl. Springer-Verlag, Berlin

Yanagimachi R (1988) Mammalian fertilization. In Physiology of Reproduction, Vol, 1, pp 135-185 Eds E Knobil and JD Neill. Raven Press, New York

Yudin AI, Gottlieb W and Meizel S (1988) Ultrastructural studies of the early events of the human sperm acrosome reaction as initiated by human follicular fluid Gamete Research 20 II-24 